\title{
Smoking cessation strategies and periodontal disease in young adults
}

Brian Mullally, Queens University of Belfast

\author{
Previous studies have related tobacco consumption to an increased \\ prevalence, extent and severity of periodontal destruction and to a poorer \\ response to therapy.
}

Several investigators have demonstrated that smoking can have a profound effect on the progression of alveolar bone loss. Often the gingival tissues in these individuals appear superficially normal. In the case of young adults, especially those with generalised forms of aggressive periodontitis this may result in extensive premature tooth loss.

Reports from the United Kingdom and the United States have charted a rise in the prevalence of smoking among adolescents. It is estimated that approximately $28 \%$ of adults in the United Kingdom are smokers while about $43 \%$ of young males and 36\% of females in their early twenties smoke cigarettes. In a study of young adults in Northern Ireland we reported that around $70 \%$ of those with generalised aggressive periodontitis were smokers, which was approximately twice the population value for comparable age groups.

Other research has indicated that the effects of tobacco are dose related and that the risk of having periodontitis increases from about threefold in those smoking less than ten a day to about six times in smokers who consume more than 30 cigarettes daily. The risk of experiencing periodontal destruction in young adults has been estimated to increase from about 6 to as much as 14 times for smokers compared with non-smokers.

The emphasis in behavioural management as it relates to the treatment of periodontal disease has traditionally been placed on plaque control. While there is no reason to reduce our efforts to improve oral hygiene in our patients there is a significant need also to address the issue of smoking cessation in the management of periodontitis. This is especially so in the case of young adults with periodontal problems as it would seem prudent to tar- get those smokers who have only recently taken up the habit.

Current recommendations for smoking cessation and the Primary Healthcare team advise the following:

- That we assess the smoking sta tus of our patients at every opportunity

- To-advise all smokers to stop

- Assist those interested in doing so

- To offer follow up

- To refer to specialist cessation services where necessary.

Although a brief intervention by health professionals may result in successful quitting by only 3\% of those addressed after one year, it has been estimated that this to expect that such advice could be delivered while waiting for a local anaesthetic to work or for crown and bridge cement to set.

In summary an effective strategy to address smoking habits in the young patient presenting with periodontitis could include:

- A widely disseminated health promotion campaign directed at the education of young individuals on the periodontal effects of cigarette smoking

- Documentation of smoking histories as a routine in patient's notes

- Training of dental professionals in both smoking cessation counselling and on the indications and use of Nicotine

\section{The dental profession has a duty to educate the public} about the relationship between smoking and the progression of periodontitis.

figure can increase to about $20 \%$ if specialist advice is reinforced with Nicotine Replacement Therapy.

The dental literature is replete with data from numerous studies, all of which conclude that smoking is a very significant factor associated with deteriorating periodontal health. For this reason the introduction of smoking cessation training into the curriculum at many dental schools and in the education of dental hygienists has become commonplace. As dentists we are well placed to reinforce these health promotional messages.

Although more prolonged sessions for counselling may be desirable the time needed for a medical practitioner to deliver smoking cessation advice is about 3 minutes. For dentists it would seem reasonable
Replacement Therapy.

- Integration of smoking cessation advice into general clinical practice.

The economic benefits of smoking cessation interventions are said to compare favourably with those gained from many other life preserving medical interventions. Research efforts continue to search for a causative link between periodontitis and heart disease. It is known that smoking is a shared risk factor for both and the dental profession has a duty to educate the public about the relationship between smoking and the progression of periodontitis. By doing so the professional advice we give to our patients may have a more significant outcome than just improved oral health. 